\title{
Esophageal tuberculosis in a patient with acquired immune deficiency syndrome: a rare presentation
}

Esophageal tuberculosis is rare and is usually secondary to infection of adjacent anatomical sites, such as the lungs [1] or mediastinal lymph nodes [1,2]; it has occasionally been reported as a single lesion [3]. There have been a few reports in the literature of esophageal tuberculosis in patients with acquired immune deficiency syndrome (AIDS) [4,5]. We report a case of histologically proved esophageal tuberculosis occurring in an AIDS patient, where this was the only manifestation of tuberculosis. We obtained written informed consent from the patient to present the case.

A 37-year-old man with AIDS and alcoholic hepatic disease presented with dysphagia, odynophagia, and vomiting for the past 1 month. His chest radiograph was normal and abdominal ultrasound revealed an enlarged periaortic lymph node. Esophagogastroduodenoscopy showed a depressed lesion with a granular fundus affecting the entire circumference of the esophagus, extending to the cardia. This lesion had a papillomatous appearance and a friable surface. A superficial ulcer, $3 \mathrm{~cm}$ in diameter, was located close to the cardia in the posterior wall, extending to the gastric body. There was thick fibrin in the fundus, and the ulcer had irregular borders, with signs of infiltration (Figure 1). Histopathological examination showed a chronic, tuberculoid, granulomatous inflammatory process and Ziehl-Neelsen staining revealed acid- and alcohol-fast bacilli, suggestive of Mycobacterium (Figure 2 ). The patient was started on tuberculostatic drugs and his symptoms were relieved, but 1 month later he developed acute intestinal obstruction and he died immediately after undergoing surgery to relieve the obstruction.

In this case the esophagus was possibly affected by contiguous spread from lymph nodes. The intestinal obstruction might have been caused by a pre-existing abdominal lesion which progressed to fibrosis and stenosis in the ileocecal region after the antituberculous treatment had
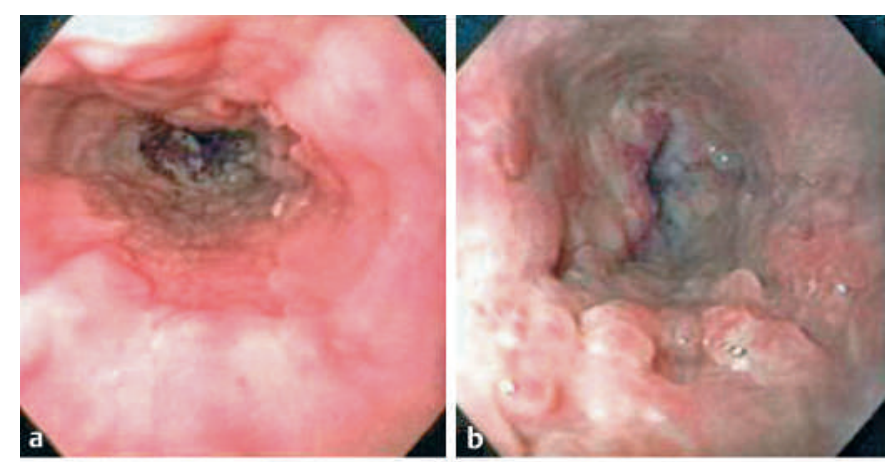

Figure 1 Endoscopic views of the esophagus and stomach, showing a depressed circumferential lesion of the esophagus, $33 \mathrm{~cm}$ from the upper dental arch (a); nodularities in the distal esophagus (b); a juxtacardiac gastric ulcer (c); and extension of the juxtacardiac gastric ulcer through to the lesser curvature of the upper stomach (d).
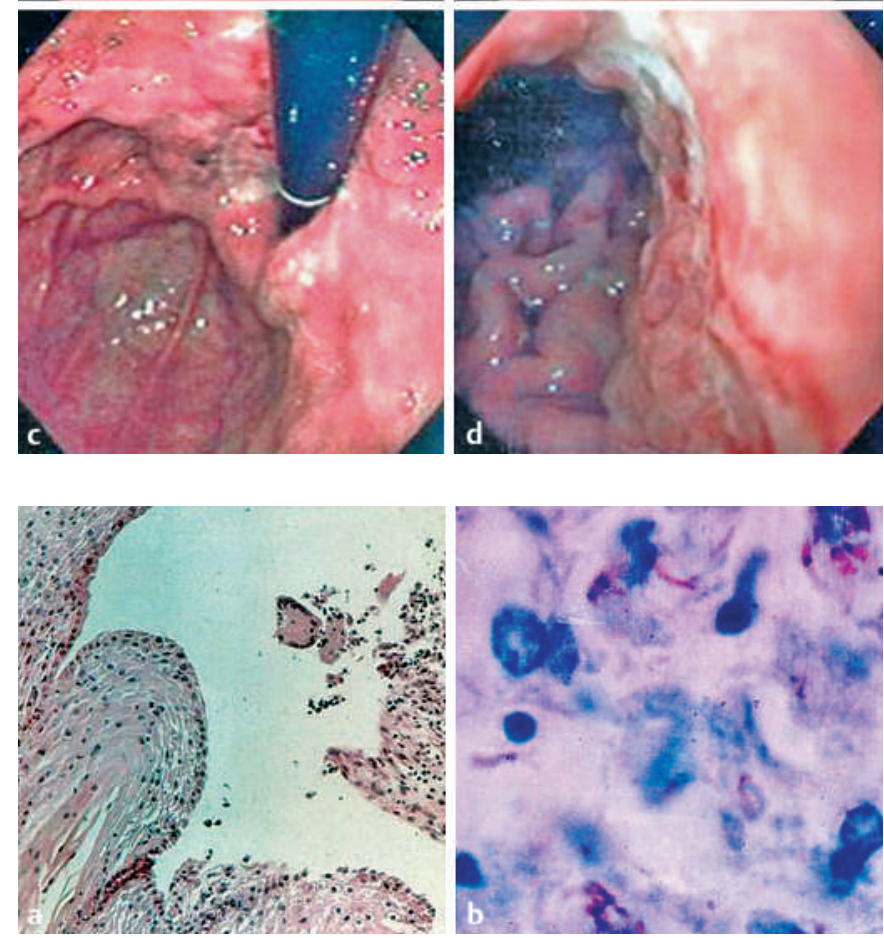

Figure 2 Histopathological examination of the esophageal ulcer showed evidence of a chronic granulomatous inflammatory process in the submucosa on hematoxylin and eosin staining (a); and the presence of acid- and alcohol-fast bacilli with morphological characteristics of Mycobacterium on Ziehl-Neelsen staining (b).

been initiated. Although esophageal involvement by tuberculosis is rare, especially in the absence of a pulmonary lesion, this possibility should be considered in the differential diagnosis of esophageal ulcers in patients with AIDS.

Endoscopy_UCTN_Code_CCL_1AB_2AC_3AZ 


\section{G. Castro', J. L. P. Módena ${ }^{2}$,}

\section{J. F. C. Figueiredo', R. Martinez ${ }^{1}$}

${ }^{1}$ Division of Tropical and Infectious

Diseases, Department of Internal

Medicine, Faculty of Medicine of

Ribeirão Preto, University of São Paulo,

Ribeirão Preto, São Paulo, Brazil

2 Department of Surgery and Anatomy, Faculty of Medicine of Ribeirão Preto, University of São Paulo, Ribeirão Preto, São Paulo, Brazil.
References

${ }^{1}$ Nagi B, Lal A, Kochhar $R$ et al. Imaging of esophageal tuberculosis: a review of 23 cases. Acta Radiol 2003; 44: 329-333

2 Fugiwara Y, Osugi H, Takada N et al. Esophageal tuberculosis presenting with an appearance similar to that of carcinoma of the esophagus. J Gastroenterol 2003; 38: 477481

${ }^{3}$ Rovekamp BT, Van der Linde K, Dees J et al. A solitary tuberculosis ulcer in the oesophagus. Eur J Gastroenterol Hepatol 2005; 17: 435-439

${ }^{4}$ Eng J, Sabanathan S. Tuberculosis of the esophagus. Dig Dis Sci 1991; 36: 536-540

${ }^{5}$ Silva R, Stoopack PM, Raufman J. Esophageal fistulas associated with mycobacterial infection in patients at risk for AIDS. Radiology 1990; 175: 449-453
Corresponding author

\section{G. Castro, M.D.}

Divisão de Doenças Infecciosas e Tropicais Departamento de Clínica Médica

Faculdade de Medicina de Ribeirão Preto Universidade de São Paulo

Av. Bandeirantes 3900

14048-900 Ribeirão Preto

São Paulo

Brasil

Fax: $\quad+55-16-3633-6695$

E-mail: gcastro@hcrp.fmrp.usp.br 